\title{
Near degeneracy and pseudo Jahn-Teller effects in mixed-valence ladders: the phase transition in $\mathrm{NaV}_{2} \mathrm{O}_{5}$
}

\author{
L. Hozoi, ${ }^{1}$ S. Nishimoto, ${ }^{2}$ and A. Yamasaki ${ }^{1}$ \\ ${ }^{1}$ Max-Planck-Institut für Festkörperforschung, Heisenbergstrasse 1, 70569 Stuttgart, Germany \\ ${ }^{2}$ Institut für Theoretische Physik, Göttingen Universität, \\ Friedrich-Hund-Platz 1, 37077 Göttingen, Germany
}

(Dated: November 27, 2018)

\begin{abstract}
We analyze the electronic structure of a mixed-valence ladder system. We find that structural anisotropy and complex electron correlations lead to on-rung charge localization and insulating character. Charge fluctuations within the rung of the ladder interact strongly to the lattice degrees of freedom, which gives rise to large pseudo Jahn-Teller effects. The phase transition in $\mathrm{NaV}_{2} \mathrm{O}_{5}$ should be driven by this kind of mechanism.
\end{abstract}

Stoichiometric compounds in which the formal valence state of an element is fractional are referred to as mixedvalence (MV) systems. MV materials display remarkable properties, such as ferrimagnetism and a metalsemiconductor transition in magnetite, a transition from a ferromagnetic metal to an antiferromagnetic insulating state in some half-doped manganites or the insulator-insulator transition towards a spin-gapped state in $\mathrm{NaV}_{2} \mathrm{O}_{5}$. The phase transitions in these compounds are often discussed in terms of charge ordering $(\mathrm{CO})$ at the transition metal (TM) sites. In such models, electrostatic interactions should yield at low temperature (LT) an ordered arrangement of the extra $d$ electrons, those associated in the ionic picture with fractional oxidation states for the TM cations. For $\mathrm{NaV}_{2} \mathrm{O}_{5}$ it was initially believed that charge order is present at all temperatures, in the form of alternating chains of $\mathrm{V}^{4+}$ and $\mathrm{V}^{5+}[1]$. However, it was later found that above $34 \mathrm{~K}$ the vanadiums are all equivalent [2, 3] and some kind of $\mathrm{CO}$ should only occur in the LT phase, presumably with a zigzag arrangement of the $d$ electrons $[4,5,6]$. In this Letter we investigate electron-lattice interactions in $\mathrm{NaV}_{2} \mathrm{O}_{5}$. We take explicitly into account the ligand degrees of freedom and find that most probably the phase transition at low temperature is induced by collective, pseudo Jahn-Teller (JT) effects involving TM- $3 d$ and oxygen $-2 p$ hole states localized within $\mathrm{V}-\mathrm{O}-\mathrm{V}$ clusters. Our findings indicate different physics as compared to the commonly accepted $d-d$ CO model. Similar JT type effects, we believe, may be relevant for other MV or doped TM materials.

Corner-sharing $\left[\mathrm{VO}_{5}\right]$ pyramids form two-leg ladders in $\mathrm{NaV}_{2} \mathrm{O}_{5}$. Such ladders are assembled in quasi twodimensional layers, with pyramids on adjacent ladders sharing edges of their bases. Interatomic interactions are strongly anisotropic, as shown by first principles bandstructure calculations [2, 7, 8]. This is related to obvious structural features: $\mathrm{V}-\mathrm{O}-\mathrm{V}$ bond angles of approximately $90^{\circ}$ between adjacent ladders, shorter $\mathrm{V}-\mathrm{O}$ bonds across the rung than along the leg, and tilting of the $\left[\mathrm{VO}_{5}\right]$ pyramids towards the rung bridging oxygen [3]. The insulating character is associated with this rungleg anisotropy and relatively large inter-rung Coulomb interactions [2, 5, 6, 7, 8, 9, 10]. In a first approximation a single $d$ electron resides on each rung, in an essentially non-bonding $d_{x y}-d_{x y}$ orbital, where the $x$ axis is parallel to the rung and $y$ is along the leg [2, 7]. Alternatively, in a configuration-interaction like picture, a superposition of left and right occupied orbital states, $d_{L, x y}^{1} d_{R, x y}^{0}$ and $d_{L, x y}^{0} d_{R, x y}^{1}$, would correspond to each $\mathrm{V}-\mathrm{O}-\mathrm{V}$ rung. If the energy difference between the $\mathrm{V} 3 d$ and $\mathrm{O} 2 p$ levels is not too large, $\mathrm{O} 2 p^{5}$ configurations may contribute as well to the ground-state (GS) wave-function. The ligand-hole configurations with the largest weight would imply holes "localized" on the rung oxygen $\mathrm{O}_{\mathrm{R}}, d_{x y}^{1} p_{y}^{1} d_{x y}^{1}$, due to strongly anisotropic $d_{x y}-p_{x, y}$ overlap integrals and lower Madelung potential at the $\mathrm{O}_{\mathrm{R}}$ site as compared to the leg oxygens $\mathrm{O}_{\mathrm{L}}[11]$. Density functional calculations indicate an energy difference $\Delta=\epsilon_{d}-\epsilon_{p} \approx 4 \mathrm{eV}$ between the $\mathrm{V} d_{x y}$ and $\mathrm{O}_{\mathrm{R}} p_{y}$ levels. However, neglecting inter-site Coulomb interactions, the on-site electron repulsion should reduce this number to $\Delta_{\text {eff }}=\Delta-U_{p p}$ for $p^{6} d^{0} \rightarrow p^{5} d^{1}$ charge transfer. Due to the extended nature of the oxygen $2 p$ functions there is no unambiguous procedure of estimating the Coulomb repulsion $U_{p p}$ between two electrons in such an orbital. Nevertheless, semiquantitative estimates in copper oxides suggest a value as high as $4 \mathrm{eV}$ [12].

In Table I we reproduce hopping matrix elements and orbital energies describing the $d_{x y}-p_{x, y}$ and $p_{x, y}-p_{x, y}$ bonding on the $\mathrm{V}-\mathrm{O}$ ladder. These were obtained by applying a powerful technique, the $N$ th-order muffin-tin or-

TABLE I: In-plane NMTO orbital energies and hoppings for the high-temperature structure of $\mathrm{NaV}_{2} \mathrm{O}_{5}(\mathrm{eV})$. $\mathrm{O}_{\mathrm{L}}^{\prime}$ stands for an oxygen ion on the leg of an adjacent ladder. For the DMRG calculations we used $\epsilon_{y}^{l}=\epsilon_{x}^{l}=-4.9$.

\begin{tabular}{cl}
\hline \hline$p d$ and $p p$ hoppings & Orbital energies \\
\hline $\mathrm{V} d_{x y}-\mathrm{O}_{\mathrm{R}} p_{y}: t_{r}=1.3$ & $\mathrm{~V}: \epsilon_{x y}=0$ \\
$\mathrm{~V} d_{x y}-\mathrm{O}_{\mathrm{L}} p_{x}: t_{l}=0.8$ & $\mathrm{O}_{\mathrm{R}}: \epsilon_{y}^{r}=-3.5$ \\
$\mathrm{~V} d_{x y}-\mathrm{O}_{\mathrm{L}}^{\prime} p_{y}: t_{l}^{\prime}=0.7$ & $\mathrm{O}_{\mathrm{L}}: \epsilon_{x}^{l}=-4.9$ \\
$\mathrm{O}_{\mathrm{R}} p_{y}-\mathrm{O}_{\mathrm{L}} p_{x}: t_{r l}=0.4$ & $\mathrm{O}_{\mathrm{L}}: \epsilon_{y}^{l}=-5.2$ \\
$\mathrm{O}_{\mathrm{L}} p_{x}-\mathrm{O}_{\mathrm{L}}^{\prime} p_{y}: t_{l l}=0.3$ & \\
\hline $\mathrm{V} d_{x y}-\mathrm{O}_{\mathrm{L}} p_{y}: t=0.3$ & \\
$\mathrm{O}_{\mathrm{R}} p_{y}-\mathrm{O}_{\mathrm{L}} p_{y}: t=0.2$ & $\mathrm{O}_{\mathrm{L}} p_{y}-\mathrm{O}_{\mathrm{L}}^{\prime} p_{y}: t=0.5$ \\
$\mathrm{O}_{\mathrm{L}} p_{x}-\mathrm{O}_{\mathrm{R}} p_{x}: t=0.2$ & $\mathrm{O}_{\mathrm{L}} p_{x}-\mathrm{O}_{\mathrm{L}}^{\prime} p_{x}: t=0.5$ \\
\hline \hline
\end{tabular}


bital (NMTO) downfolding method recently developed in Stuttgart [13]. In order to generate an effective Hamiltonian capable of modeling metal-ligand interactions, we downfold all basis functions other than $\mathrm{V} d_{x y}$ and $\mathrm{O} 2 p$.

We use the NMTO hoppings and orbital energies to investigate the nature of the many-electron GS wavefunction of a V-O Hubbard type ladder model. The Hamiltonian is written as 10, 15]:

$$
\begin{aligned}
H & =\epsilon_{d} \sum_{i \sigma} n_{i \sigma}+\sum_{j \sigma} \epsilon_{p}^{j} n_{j \sigma}+\sum_{<i j>\sigma} t_{p d}^{j}\left(d_{i \sigma}^{\dagger} p_{j \sigma}+\text { h.c. }\right) \\
& +\sum_{<j j^{\prime}>\sigma} t_{p p}^{j j^{\prime}}\left(p_{j \sigma}^{\dagger} p_{j^{\prime} \sigma}+\text { h.c. }\right)+U_{d d} \sum_{i} n_{i \uparrow} n_{i \downarrow} \\
& +U_{p p} \sum_{j} n_{j \uparrow} n_{j \downarrow}+V_{p d} \sum_{<i j>\sigma \sigma^{\prime}} n_{i \sigma} n_{j \sigma^{\prime}} .
\end{aligned}
$$

We neglect the apical ligands and restrict to a single orbital at each site: $\mathrm{V} d_{x y}, \mathrm{O}_{\mathrm{R}} p_{y}, \mathrm{O}_{\mathrm{L}} p_{x}$ on the same ladder plus nearest neighbor (n.n.) $\mathrm{O}_{\mathrm{L}}^{\prime} p_{y}$ orbitals on adjacent ladders. We also neglect the finite $\mathrm{V} d_{x y}-\mathrm{O}_{\mathrm{L}} p_{y}$ overlap, which should be zero for $\mathrm{V}$ and $\mathrm{O}_{\mathrm{L}}$ atoms in the same $z y$ plane, and $\pi$-type $p p$ hoppings like those listed in the lower part of Table I. Still, we believe that our model is able to capture the essential properties of $\mathrm{V}-\mathrm{O}$ ladders in $\mathrm{NaV}_{2} \mathrm{O}_{5}$. GS expectation values are obtained by density-matrix renormalization-group (DMRG) methods [14]. For these calculations up to 20 rungs on the $\mathrm{V}-\mathrm{O}$ ladder, open-end boundary conditions, and up to $m=2000$ states to build the DMRG basis were used. The typical discarded weight was $10^{-11}-10^{-10}$. We employed the code developed by Jeckelmann et al. 15]. The results reported here were obtained with $U_{d d}=3 \mathrm{eV}$, as deduced in [2, 7, 8 ], and the same $U_{p p}$ for $\mathrm{O}_{\mathrm{R}}$ and $\mathrm{O}_{\mathrm{L}}$.

For reasonable values of the $U_{p p}$ and $V_{p d}$ parameters, the DMRG results show significant $\mathrm{O}_{\mathrm{R}}$ hole character for the GS wave-function, see Fig.1(a). With $U_{p p}=4 \mathrm{eV}$ and $V_{p d}=0.25 \mathrm{eV}$, for example, the occupation of the rung $p_{y}$ orbital is 1.60 , which indicates that the overall weights of the $\mathrm{O}_{\mathrm{R}} p_{y}^{2}$ and $p_{y}^{1}$ configurations are nearly equal. Yet the ladder is insulating, with a finite charge transfer gap, see Fig.1(b). The fact that the gap is strongly dependent on the rung - leg anisotropy was previously evidenced in [10]. Regarding the other parameters, the most important is $U_{p p}$ in our model. In simple words, for a given $V_{p d}$, the size of $U_{d d}$ "matters" when $U_{p p}$ is sufficiently high to give large occupation at the $d$ sites.

Physical intuition would point to a strong electronlattice coupling for bond-stretching vibrations within the $\mathrm{V}-\mathrm{O}_{\mathrm{R}}-\mathrm{V}$ unit. The situation should be somewhat similar to the in-plane $\mathrm{O}$ half-breathing mode in doped copper oxides, see for example [16] and Refs. therein. To study such couplings in the MV ladder, we simulate the displacement of the rung ligands by introducing a modulation of the rung transfer integrals, $t_{r} \sim r^{-7 / 2}$ [17], and inter-site Coulomb repulsion, $V_{p d}^{r} \sim 1 / r$. The effect of these displacements on the GS (electronic) energy is investigated by DMRG calculations. However, before discussing the DMRG results, we illustrate the nature of the
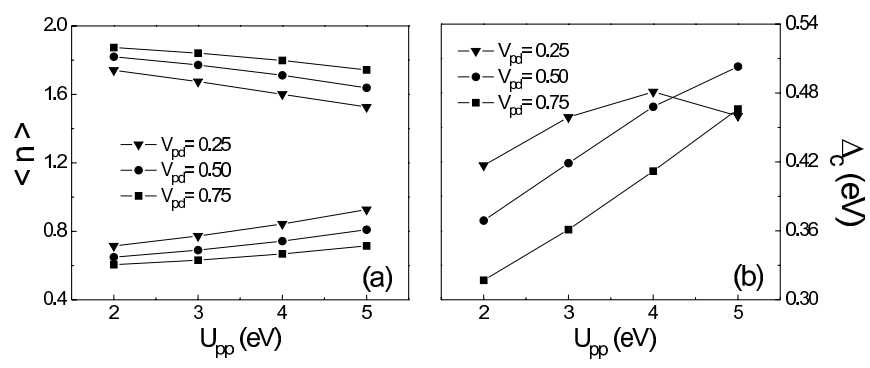

FIG. 1: (a) Occupation of the $\mathrm{V}_{x y}$ and $\mathrm{O}_{\mathrm{R}} p_{y}$ orbitals. Up to the 2nd digit these numbers are constant along the ladder. For $\mathrm{O}_{\mathrm{L}} p_{x}$ and $\mathrm{O}_{\mathrm{L}}^{\prime} p_{y}$ the occupation numbers are not less than 1.85. Results with $L=12$ rungs and $m=1600$ DMRG states. (b) Charge gap $\Delta_{c}$ for ladders with $L=12$ rungs and $3 L$ holes, where $\Delta_{c}=1 / 2[E(3 L+2)+E(3 L-2)-2 E(3 L)]$. The values extrapolated to $1 / L \rightarrow 0$ through $1 / L=1 / 16$ and $1 / L=1 / 20$ change by $15-25 \%$, with larger differences for higher $U_{p p}$.

electron - lattice interactions on an oversimplified, singlerung model. We first assume that in $\mathrm{NaV}_{2} \mathrm{O}_{5}$ three electrons (holes) are "localized" on each $d_{x y}-p_{y}-d_{x y}$ "rung". For an isolated 3-site rung, we deduce then the expression for the lowest energy eigenvalue corresponding to a superposition of $p_{y}^{2}$ and $p_{y}^{1}$ configurations. With $V_{p d}=0$, $\Delta_{\text {eff }}=\Delta-U_{p p}$, and neglecting double occupation at the $d$ sites, the Hamiltonian reads in matrix notation

$$
H_{r}=\left(\begin{array}{ccccc}
0 & 0 & 0 & t_{R} & t_{R} \\
0 & 0 & t_{L} & 0 & t_{L} \\
0 & t_{L} & \Delta_{\mathrm{eff}} & 0 & 0 \\
t_{R} & 0 & 0 & \Delta_{\mathrm{eff}} & 0 \\
t_{R} & t_{L} & 0 & 0 & \Delta_{\mathrm{eff}}
\end{array}\right),
$$

where $t_{L, R}(x) \approx t_{r} /(1 \pm x)^{7 / 2}, x=\delta x / r\left(\mathrm{VO}_{\mathrm{R}}\right), \delta x$ is the displacement of the $\mathrm{O}_{\mathrm{R}}$ ion along the rung direction, and $r\left(\mathrm{VO}_{\mathrm{R}}\right) \approx 1.83 \AA[3]$. The lowest eigenstate is a doublet and has an energy $E=1 / 2 \Delta_{\text {eff }}-1 / 2\left(\Delta_{\text {eff }}^{2}+4 t_{L}^{2}+4 t_{R}^{2}+\right.$ $\left.4\left(t_{L}^{4}+t_{R}^{4}-t_{L}^{2} t_{R}^{2}\right)^{1 / 2}\right)^{1 / 2}$. We express $E(x)$ as an onedimensional (1D) series expansion and obtain:

$$
E(x)=E(0)-\frac{1}{4} \frac{630 t_{r}^{2}}{\left(\Delta_{\text {eff }}^{2}+12 t_{r}^{2}\right)^{1 / 2}} x^{2}+O\left(x^{4}\right) .
$$

We find thus a negative, "electronic" contribution to the harmonic force constant associated with this displacement. If we take the values from Table I, $t_{r}(0)=t_{r}=1.3$ and $\Delta=3.5$, choose $U_{p p}=3 \mathrm{eV}$, express the energy in units of $\mathrm{meV}$ and the distortion in units of percents of the $\mathrm{VO}_{\mathrm{R}}$ bond length, we find $E(x)-E(0) \approx 1 / 2 k_{\mathrm{el}} x^{2}$, $k_{\mathrm{el}}=-11.7$. This is a huge amount, about $35 \mathrm{eV} / \AA^{2}$. For the isostructural $\mathrm{CaV}_{2} \mathrm{O}_{5}$ compound, a $3 d^{1}$ Mott insulator [18], the infrared active $\mathrm{VO}_{\mathrm{R}}$ bond-stretching phonon was associated to the $B_{3 u}$ mode at $515 \mathrm{~cm}^{-1}$ [19], which gives a force constant $k_{\mathrm{ir}}^{\prime}=15.6 \mathrm{eV} / \AA^{2}$. For $\mathrm{NaV}_{2} \mathrm{O}_{5}$, the appearance of continuum features in this frequency range, in both reflectivity $B_{3 u}$ and Raman $B_{2 g}$ spectra, makes the interpretation difficult [19, 20]. Nevertheless, this continuum proves the existence of strong electronlattice interactions. 
The large magnitude of this effect is confirmed by DMRG calculations on the $\mathrm{V}-\mathrm{O}$ Hubbard ladder, although more complex interactions are now involved. Contributions to the harmonic force constant induced by electron-lattice couplings are plotted for various $U_{p p}$ and $V_{p d}$ values in Fig. 2(a). The numbers were obtained by introducing an in-line pattern of (static) $\mathrm{V}-\mathrm{O}_{\mathrm{R}}-\mathrm{V}$ distortions. Each $\mathrm{O}_{\mathrm{R}}$ was displaced by the same amount and all other ions were kept fixed. To extract the $k_{\text {el }}$ parameters we calculated $E(x)$ for several small $x$ values. We also considered the bending of the $\mathrm{V}-\mathrm{O}_{\mathrm{R}}-\mathrm{V}$ bond.

Effects induced by the vibronic mixing of two or several electronic states are referred to as (pseudo) JT effects 21]. The presence of quasilocalized and relatively close in energy states plus the strong coupling between the electronic and nuclear motion in $\mathrm{NaV}_{2} \mathrm{O}_{5}$ can be included then within this class of phenomena. We checked for the existence of similar effects in $\mathrm{CaV}_{2} \mathrm{O}_{5} \cdot \mathrm{CaV}_{2} \mathrm{O}_{5}$ is also highly anisotropic 22]. However, the nature of the ground and low-lying excited states is different and also their energy separation is larger, not less than $U_{d d}$. Such interactions are expected here to be weaker, which is indeed confirmed by DMRG calculations on a "halffilled" $d^{1}-p^{6}-d^{1}$ ladder. For $U_{d d}=3 \mathrm{eV}[18], 2 \leq U_{p p} \leq 5$, and $0.25 \leq V_{p d} \leq 0.75$ the electronic energy per single rung varies as $E(x)-E(0) \approx 1 / 2 k_{\mathrm{el}}^{\prime} \delta x^{2}, 2.4<-k_{\mathrm{el}}^{\prime}<7.0$ $\left(\mathrm{eV} / \AA^{2}\right)$. Although less drastic, this still should produce some phonon softening. We obtain thus a "bare" harmonic force constant $k^{\prime}=k_{\mathrm{ir}}^{\prime}+\left|k_{\mathrm{el}}^{\prime}\right|$. We assume now that $k^{\prime}$ is also a reasonable estimate for the bare harmonic force constant in $\mathrm{NaV}_{2} \mathrm{O}_{5}$ and display in Fig. 2(b) an overall force constant $A=k^{\prime}-\left|k_{\mathrm{el}}\right|$ that should define the 2nd order term in the 1D Taylor expansion of the potential energy. We see that for certain values of $U_{p p}$ and $V_{p d} A$ is negative, which shows that the system is instable with respect to such deformations. The finite amplitude of these displacements is related to the presence of higher order, positive terms. In the simplest approximation we arrive at the so-called $\phi^{4}$ model, widely used to model local anharmonic effects in insulators [23]: $V_{R}=1 / 2 A x^{2}+1 / 4 B x^{4}$.

Near degeneracy and a large contribution of oxygenhole configurations to the GS wave-function were actually predicted by multiconfiguration, quantum chemical calculations on small clusters 24, 25]. Even more, these $a b$ initio calculations [25] indicate a double-well potential for distortions of the rung oxygen along the $x$ axis and give strong support for the model we propose here. Whereas the approximate treatment of so-called dynamic electron correlation effects might somewhat overestimate the weight of the $\mathrm{O}_{\mathrm{R}} p_{y}^{1}$ configuration and cannot guarantee a highly accurate value for the $A$ parameter, we still can extract from the results of [25] a good estimate for $B$, essentially determined by "hard" core-core repulsion. Using the units of Fig. $2(\mathrm{~b})$ and $V_{R}^{\text {min. }}=V_{R}\left(x_{0} \approx 3 \%\right)=-42 \mathrm{~K}$ [25], we find $B=4 V_{R}^{\text {min. }} / x_{0}^{4}=0.18$. The DMRG results show a 4 th order term in the purely electronic $E(x)$ expansion of the order of $1 \%$ of this value. For the bond-

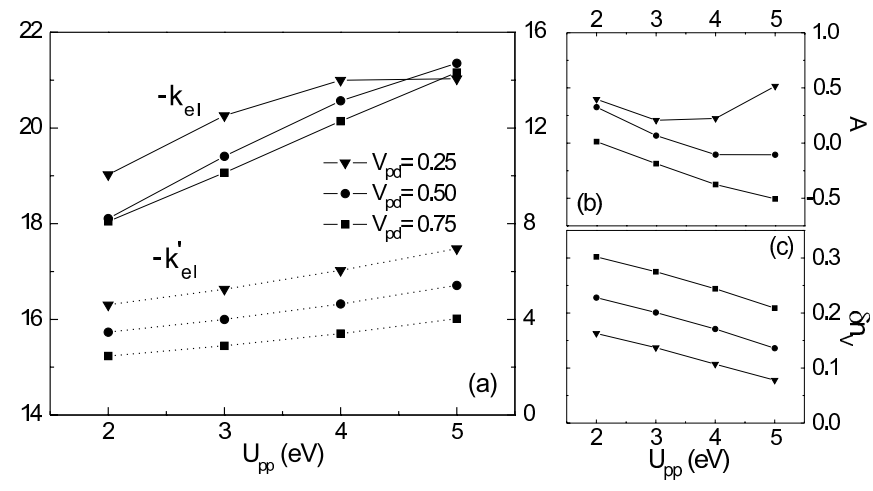

FIG. 2: (a) $k_{e l}$ (full lines, left scale) and $k_{e l}^{\prime}$ (dots, right scale) parameters describing the effect of rung distortions on the electronic energy per rung of the $\mathrm{V}-\mathrm{O}$ ladder in $\mathrm{NaV}_{2} \mathrm{O}_{5}$ and $\mathrm{CaV}_{2} \mathrm{O}_{5}\left(\mathrm{eV} / \AA^{2}\right)$. (b) $A$ parameter associated with the $\phi^{4}$ model, see text. Units of meV for energy and percents of the $\mathrm{VO}_{\mathrm{R}}$ bond length for the amplitude of the distortion were used. (c) $\mathrm{V}-\mathrm{V}$ charge disproportion $\delta n_{V}, \delta n_{V}=n\left(d_{L, x y}\right)-$ $n\left(d_{R, x y}\right)$, for a zigzag arrangement of the rung oxygens with $x=\delta x / r\left(\mathrm{VO}_{\mathrm{R}}\right)= \pm 3 \%$. In each figure $L=12, m=1600$, and the same symbols were used to plot the curves.

stretching vibration of the $\mathrm{O}_{R}$ ion we can also evaluate inter-rung couplings. We model these oxygens by simple point charges and consider only $\mathrm{O}_{R}-\mathrm{O}_{\mathrm{R}}$ Coulomb interactions. To lowest order we find a n.n. interaction

$$
\begin{aligned}
V_{R R} & \approx-\sum_{j=1}^{4} \frac{C_{a}}{2}\left(x_{i}-x_{j}^{a}\right)^{2} \\
& -\sum_{k=1}^{2} \frac{C_{b}}{2}\left(x_{i}-x_{k}^{b}\right)^{2}-\sum_{l=1}^{2} \frac{C_{c}}{2}\left(x_{i}-x_{l}^{c}\right)^{2},
\end{aligned}
$$

where $C_{b}=q^{2} r\left(\mathrm{VO}_{\mathrm{R}}\right)^{2} / b^{3}$ and $C_{c}=q^{2} r\left(\mathrm{VO}_{\mathrm{R}}\right)^{2} / c^{3}$ are coupling constants within the $b c$ plane and a slightly more complicated expression corresponds to the inter-ladder coupling $C_{a}$ in the $a b$ plane. With an "effective" charge for the $\mathrm{O}_{\mathrm{R}}$ ion $q \approx 1.5$ and the lattice constants from [3], these inter-rung couplings are $C_{a}=0.19, C_{b}=0.23$, $C_{c}=0.10$. We note here that the DMRG GS energy for a zigzag configuration of the $\mathrm{O}_{\mathrm{R}}$ oxygens is always slightly lower than the energy for in-line displacements. This would give some extra contribution to the $C_{b}$ parameter. Parametrized Hamiltonians based on low-order Taylor expansions of the energy surface were intensively studied in connection with structural phase transitions in ferroelectrics, by both molecular-dynamics and Monte Carlo simulations 26, 27, 28]. Whereas such a numerical study is beyond the scope of this paper, we still can make a rough estimate of the transition temperature. In a mean field treatment $T_{c} \sim(2 d \tilde{C}) A / B$ [23], where $d$ is the system's dimension and $\tilde{C}$ is an isotropic n.n. coupling. Although not accurate, this gives at least the order of magnitude of $T_{c}$, as comparison to numerical results shows [26]. For $U_{p p}=5$ and $V_{p d}=0.75, A=-0.51$ and with $\tilde{C} \approx 0.18$ we obtain $T_{c} \approx 47 \mathrm{~K}$. We note, however, 
that a number of other effects were neglected, such as charge polarization, other $p d$ and $p p$ hoppings, longer range electrostatic interactions, coupling to other modes, e.g. the $\mathrm{V}-\mathrm{O}_{\mathrm{R}}-\mathrm{V}$ bending, and coupling to the strain. The last effects should determine the actual LT crystal structure.

We turn now to the analysis of the GS wave-function with displaced $\mathrm{O}_{\mathrm{R}}$ ions and plot in Fig. 2(c) the changes induced by rung distortions on the $\mathrm{V} d_{x y}$ orbital occupation numbers. For the $U_{p p}$ and $V_{p d}$ values considered here, the on-rung $\mathrm{V}-\mathrm{V}$ charge disproportion does not exceed 0.31 for $x=3 \%$. The values would probably decrease somewhat when including on-rung polarization effects. Recent interpretations of the experimental data 29] seem to agree with our results.

In conclusion, we investigated the interplay between electron and lattice degrees of freedom for a MV ladder system. We found that structural anisotropy and complex Coulomb correlations lead to quasilocalized, threehole states on each rung. Charge fluctuations within such an "unsaturated" metal $3 d-\mathrm{O} 2 p$-metal $3 d$ structure are coupled to the lattice, which gives rise to strong JT type interactions. We suggest that the phase transition in
$\mathrm{NaV}_{2} \mathrm{O}_{5}$ is driven by cooperativity among these on-rung JT effects. We mapped results of DMRG calculations for a $p-d$ Hubbard type ladder onto a $\phi^{4}$ model. For reasonable values of the on-site and inter-site electron repulsion parameters we could reproduce in a mean field approximation the order of magnitude of the transition temperature. The spin-gap formation below $34 \mathrm{~K}$ is probably related to the existence of different $\mathrm{V}-\mathrm{O}_{\mathrm{L}}-\mathrm{V}$ paths between consecutive rungs on the same ladder [6, 25, 30]. The dielectric anomalies at $T_{c}$ [4] should be induced by zigzag ion ordering and only small $\mathrm{V}-\mathrm{V}$ charge disproportion. We also mention that for certain values of the Coulomb repulsion parameters, see Fig. 1, the GS wavefunction has significant oxygen-hole character. Strong contributions of oxygen-hole configurations were recently proposed for other MV oxides, such as the half-doped manganites, see 31] and Refs. therein.

We thank J. Kortus, T. Saha-Dasgupta, M. Mostovoy, O. Jepsen, O. K. Andersen, and O. Gunnarsson for help at various stages of this work and stimulating discussions. L. H. acknowledges financial support from the Alexander von Humboldt Foundation.
[1] A. Carpy and J. Galy, Acta Crystallogr. B 31, 1481 (1975).

[2] H. Smolinski et al., Phys. Rev. Lett. 80, 5164 (1998).

[3] A. Meetsma et al., Acta Crystallogr. C 54, 1558 (1998); H. G. von Schnering et al., Z. Kristallogr. 213, 246 (1998); T. Chatterji et al., Solid State Commun. 108, 23 (1998).

[4] A. I. Smirnov et al., ibid. 59, 14546 (1999); M. Poirier et al., ibid. 60, 7341 (1999).

[5] H. Seo and H. Fukuyama, J. Phys. Soc. Jpn. 67, 2602 (1998).

[6] M. V. Mostovoy, D. I. Khomskii, and J. Knoester, Phys. Rev. B 65, 64412 (2002); M. V. Mostovoy and D. I. Khomskii, Solid State Commun. 113, 159 (2000).

[7] A. N. Yaresko et al., Phys. Rev. B 62, 15538 (2000).

[8] V. V. Mazurenko et al., ibid. 66, 081104 (2002).

[9] P. Horsch and F. Mack, Eur. Phys. J. B 5, 367 (1998).

[10] S. Nishimoto and Y. Ohta, J. Phys. Soc. Jpn. 67, 2996 (1998).

[11] The rung oxygen $\mathrm{O}_{\mathrm{R}}$ has only two $\mathrm{V}$ nearest neighbors, whereas the leg oxygen $\mathrm{O}_{\mathrm{L}}$ has three.

[12] A. K. McMahan, R. M. Martin, and S. Satpathy, Phys. Rev. B 38, 6650 (1988).

[13] O. K. Andersen and T. Saha-Dasgupta, ibid. 62, 16219 (2000); O. K. Andersen, T. Saha-Dasgupta, and S. Ezhov, Bull. Mater. Sci. 26, 19 (2003).

[14] See for example U. Schollwöck, Rev. Mod. Phys., in press.

[15] E. Jeckelmann, D. J. Scalapino, and S. R. White, Phys. Rev. B 58, 9492 (1998); S. Nishimoto, E. Jeckelmann, and D. J. Scalapino, ibid. 66, 245109 (2002).

[16] O. Rösch and O. Gunnarsson, ibid. 70, 224518 (2004); S.
Ishihara and N. Nagaosa, ibid. 69, 144520 (2004).

[17] W. A. Harrison, Electronic Structure and the Properties of Solids (Dover, New York, 1989).

[18] M. A. Korotin et al., J. Phys.: Condens. Matter 12, 113 (2000).

[19] Z. V. Popovíc et al., Phys. Rev. B 65, 184303 (2002).

[20] Z. V. Popovíc et al., Solid State Commun. 110, 381 (1999); W. S. Bacsa et al., Phys. Rev. B 61, 14885 (2000).

[21] I. B. Bersuker, Chem. Rev. 101, 1067 (2001); G. A. Gehring and K. A. Gehring, Rep. Prog. Phys. 38, 1 (1975).

[22] NMTO downfolding in $\mathrm{CaV}_{2} \mathrm{O}_{5}$ gives $\epsilon_{x y}=0, \epsilon_{y}^{r}=-4.0$, $\epsilon_{x}^{l}=-5.1, \epsilon_{y}^{l}=-5.4, t_{r}=1.15, t_{l}=0.9, t_{l}^{\prime}=0.8, t_{r l}=0.35$, $t_{l l}=0.3(\mathrm{eV})$.

[23] A. D. Bruce and R. A. Cowley, Structural Phase Transitions (Taylor \& Francis, London, 1981).

[24] N. Suaud and M.-B. Lepetit, Phys. Rev. Lett. 88, 056405 (2002).

[25] L. Hozoi et al., Phys. Rev. B 67, 035117 (2003).

[26] T. Schneider and E. Stoll, Ferroelectrics 24, 67 (1980).

[27] W. C. Kerr and A. R. Bishop, Phys. Rev. B 34, 6295 (1986).

[28] W. Zhong, D. Vanderbilt, and K. M. Rabe, ibid. 52, 6301 (1995).

[29] C. Presura et al., ibid. 61, 15762 (2000); Y. FagotRevurat, M. Mehring, and R. K. Kremer, Phys. Rev. Lett. 84, 4176 (2000); Y. Joly, S. Grenier, and J. E. Lorenzo, Phys. Rev. B 68, 104412 (2003).

[30] A. Bernert et al., Eur. Phys. J. B 21, 535 (2001).

[31] M. Coey, Nature 430, 155 (2004). 